\title{
Detection of duck hepatitis virus serotype 1 by biosensor based on imaging ellipsometry
}

\author{
Chenghong Huang ${ }^{\mathrm{a}, \mathrm{c}, \mathrm{e}, 1}$, Jixiang $\mathrm{Li}^{\mathrm{b}, 1}$, Yu Tang ${ }^{\mathrm{b}}$, Yanyan Chen ${ }^{\mathrm{a}}$, Gang Jin ${ }^{\mathrm{a}, \mathrm{c}, \mathrm{d}, *}$ \\ a Suzhou Institute of Nano-Tech and Nano-Bionics, CAS, Suzhou 215125, China \\ ${ }^{\mathrm{b}}$ Rongchang Campus, University of Southwest, Chongqing 402460, China \\ ${ }^{\mathrm{c}}$ Institute of Biophysics, CAS, Beijing 100101, China \\ d Key Laboratory of Microgravity, Institute of Mechanics, CAS, Beijing 100190, China \\ ${ }^{\mathrm{e}}$ Graduate School of the Chinese Academy of Sciences, Beijing, China
}

\section{A R T I C L E I N F O}

\section{Article history:}

Received 7 June 2010

Received in revised form

29 July 2010

Accepted 2 August 2010

Available online 10 August 2010

\section{Keywords:}

Biosensor

Imaging ellipsometry

Duck hepatitis virus

Detection

\begin{abstract}
A B S T R A C $T$
A novel method for the detection of duck hepatitis virus serotype1 (DHV1) using a biosensor based on imaging ellipsometry (IE) is developed. Polyclonal antibodies against DHV1 were immobilized on silicon wafers through protein A and used to capture virus particles in sample. Signals on silicon surface were acquired directly in grayscale format on an imaging ellipsometry image. This study demonstrated that the assay is rapid and accurate for detection of duck hepatitis virus. Compared with traditional methods, this technique not only has high sensitivity as low as $8 \times 10^{-9.5} \mathrm{LD}_{50} / \mathrm{ml}$, but can also simultaneously perform multiple serotype identification of DHV1, DHV1-g and DHV1-s. These results suggest that this biosensor might be a practicable diagnostic tool for duck hepatitis virus detection.
\end{abstract}

(c) 2010 Elsevier B.V. All rights reserved.

\section{Introduction}

Four types of duck hepatitis viruses [1] including type1, DHV1a (a variant strain of DHV1), DHV2 and DHV3 [2-4] can cause duck hepatitis and bring immeasurable losses to waterfowl breeding. DHV1 was first ascertained in the United States, while DHV2 and DHV3 have only been reported in the UK and the USA [5,6]. Two variant strains of DHV1 were also isolated between 2006 and 2009 from Guangdong and Sichuan province in China by the staff in our laboratory. They were designated as DHV1-g and DHV1-s, respectively. Traditional methods for DHV1 detection are based on virus isolation through tissue culture. Virus isolation is an effective method to obtain whole virus particles, but it is labor-intensive and time-consuming. Although other methods such as reverse transcription polymerase reaction (RT-PCR) etc. are used for testing of viral nucleic acids with highly sensitive and rapid diagnosis, they are inconvenient for common laboratory. Therefore, to develop a new method for DHV detection with advantages of simple and short-time is essential for clinic application.

\footnotetext{
* Corresponding author. Suzhou Institute of Nano-Tech and Nano-Bionics, CAS, Suzhou 215125, China. Tel.: +86 1082544318.

E-mail addresses: yychen2006@sinano.ac.cn (Y. Chen), gajin@imech.ac.cn (G. Jin).

${ }^{1}$ Both authors contributed equally to this work.
}

Recently, biosensors for microbe detection have received rapid development and wide applications [7,8]. The preparation of the biosensor involves the immobilization of antibodies onto solidphase surfaces. Two routine strategies including physical adsorption and covalently bonding are generally adopted for antibody immobilization. Immobilization of antibodies by physical adsorption might leads to problems associated with poor reproducibility because adsorbed antibodies will easily shed from the surface. The covalently immobilized antibodies suffer primarily from random orientations, which might somewhat decrease their efficiency of antigen binding. Therefore, special interest has focused on the development of oriented immobilization of antibodies through protein $A / G$ to specifically bind the Fc fragment of the immunoglobulin (IgG). The antibody after oriented immobilization is favorable for antigen accessible.

Biosensor based on imaging ellipsometry (BIE), first put forward by Jin [9], is a powerful tool for thin layer analysis. Previous work related to BIE has already been theoretically and empirically described in literatures [10-13]. Recently, application of BIE to microorganism and protein marker detection made substantial progresses [14,15]. High resolution of imaging ellipsometry combined with high throughput of array can also play important role in the detection of multiple serotypes of species, for instance, bacteria or virus. The purpose of this study is to specifically detect DHV1 using array. The main advantages of this array are that 
antibodies from different types through oriented immobilization are not only propitious to bind virus particles with high sensitive, but also helpful for multiplex analysis in parallel with a single experiment operation. The clinic application will be further discussed.

\section{Material and methods}

\subsection{BIE principle}

BIE has been developed in Institute of Mechanics (Chinese Academy of Sciences) for the purpose of performing parallel immunoassays and is based on a combination of an integrated microfluidic reactor array system and imaging ellipsometry (IE). The microfluidic system is used for surface patterning and array fabrication, and for solution delivery, antibody immobilization and antigen capture. IE is used for reading the protein arrays.

The microfluidic system in PDMS template contains a $6 \times 4$ cell array. When the template was attached to the silicon slide surface, 24 individual chambers were formed independently and 24 unit dots in the array were patterned onto the silicon surface. Each chamber had two access holes where solution could pass in and out of the chamber through them. By such a microfluidic system, ligand solutions were delivered individually to different cells for immobilization so that the surface is patterned homogeneously and simultaneously in array format. Furthermore, multiple samples can accordingly be analyzed simultaneously.

Imaging ellipsometry was used for the visualization and quantification of the protein adsorption layer on the surface of silicon substrate. It is a powerful tool for optical characterization of surfaces and thin films and very sensitive to significant changes in optical parameters with a sub-nanometer resolution of vertical. During experiments, the optical components in the system are adjusted to fulfill the null conditions on a silicon wafer without any adsorbed protein layers and to measure the adsorption layer thickness when the protein was adsorbed. Under this condition, the detected signal density $I$ is related to the thickness $(d)$ of the protein layer according to $I=k d^{2}$, where $k$ is a constant. The ellipsometry image was focused on the sensing area of the CCD camera. A digital image was grabbed by and stored in a computer with a grayscale format ( 8 bits, 0-255 grayscale) for further evaluation by imageprocessing software of the program.

\subsection{Materials}

The viruses and bacteria used in this study are listed in Table 1. Antisera (IgG) were collected from immunized rabbit blood and further purified for use. Silicon wafers were purchased from General Research Institute for Nonferrous Metals of China. Bovine serum albumin (BSA) was obtained from Sigma. 3-Aminopropyltriethoxysilane (APTES), 1-(3-Dimethylaminopropyl)-3-ethylcarbobiimide hydrochloride (EDC); N-hydroxy-droxysuccinimde (NHS) and succinimide anhydride were obtained from ACROS. Tween-20 was purchased from Sigma-Aldrich. Deionized water

Table 1

The reference pathogens used for the BIE detection.

\begin{tabular}{lll}
\hline Species & Strain/serotype & Host \\
\hline Duck hepatitis virus (DHV) & AV211, type1 & Peking duck \\
Duck hepatitis virus (DHV) & Field isolate, type1-g & Peking duck \\
Duck hepatitis virus (DHV) & Field isolate, type1-s & Peking duck \\
Avian influenza virus (AI) & Field isolate, H9N2 & Peking duck \\
Gosling plague virus (GPV) & Field isolate & China gosling \\
Rimerella anatipestifer (R.A.) & Field isolate, type1 & Peking duck \\
E. coli $($ E coli) & Field isolate, O157:H7 & Peking duck \\
\hline
\end{tabular}

(Resistivity $18.3 \mathrm{M} \Omega \mathrm{cm}$ ) was produced by a Millipore system (Millipore, Bedford, MA). Other heterogeneous species used are listed in Table 1.

\subsection{Substrate modification}

Silicon wafer was initially treated with piranha solution $(30 \%$ $\mathrm{H}_{2} \mathrm{O}_{2}: 70 \% \mathrm{H}_{2} \mathrm{SO}_{4}=1: 3, \mathrm{v} / \mathrm{v}$ ) for $30 \mathrm{~min}$ followed by intensive rinsing with deionized water. The oxidized silicon wafer was immersed in ethanol solution containing APTES (5\%) for $2 \mathrm{~h}$ incubation. After washed by ethanol and deionized water repeatedly, the modified silicon wafer was placed in saturated succinic anhydride solution of ethanol for $12 \mathrm{~h}$ incubation, then, washed by ethanol several times again. As such, $-\left(\mathrm{CH}_{3}\right) \mathrm{NH}-\mathrm{CO}\left(\mathrm{CH}_{2}\right)_{2}-\mathrm{COOH}$ group on wafer was generated. The prepared wafers were stored in ethanol solution at $4{ }^{\circ} \mathrm{C}$ refrigerator until use.

\subsection{IgG immobilization and DHV1 detection}

The detection configuration is illustrated in Fig. 1. When a modified silicon wafer was applied to microfluidic channel system, the carboxyl group on its surface was activated by NE solution. The NE solution was prepared with NHS/EDC solution $(0.05 / 0.02 \mathrm{M})$ in deionized water, and $50 \mu \mathrm{l}$ of NE solution was then added to the microfluidic channel system (ISMATEC, SA, Switzerland, 24-channel peristaltic pump) at a flow rate of $5 \mu \mathrm{l} / \mathrm{min}$ and passed through the surface of the wafer. Protein A $(200 \mu \mathrm{g} / \mathrm{ml})$ was added to the corresponding channel $(20 \mu \mathrm{l}, 1 \mu / \mathrm{min})$ and covalently immobilized on the silicon surface. Later, $\operatorname{IgG}(100 \mu \mathrm{g} / \mathrm{ml}$, $1 \mu \mathrm{l} / \mathrm{min}$ ) was dispensed to the channel to interact $30 \mathrm{~min}$. Next, the surface was blocked by $1.0 \mathrm{mg} / \mathrm{ml} \mathrm{BSA}(30 \mu \mathrm{l}, 1 \mu \mathrm{l} / \mathrm{min})$. The channels were rinsed with PBST $(50 \mu \mathrm{l}, 10 \mu \mathrm{l} / \mathrm{min})$. The DHV in samples (purified or clinical collected) were next added to channels for $30 \mathrm{~min}$ incubation ( $30 \mu \mathrm{l}, 1 \mu \mathrm{l} / \mathrm{min}$ ). At last, the wafer was rinsed with PBST and deionized water and taken out of the microfluidic system. After rinsing with deionized water repeatedly and drying under a stream of nitrogen, the wafer was delivered to ellipsometry imaging for grayscale measurement.

\subsection{Analytical sensitivity, specificity and reproducibility}

The sensitivity was determined by adding three standard deviations to the mean grayscale value of 10 zero standard replicates and calculating the corresponding concentration. Avian influenza virus, Gosling plague virus, Rimerella anatipestifer and Escherichia coli were added to the sensing dots to assess grayscale response [16]. Analytic reproducibility, expressed as CV\%, was conducted by evaluating intra-slide and inter-slide reproducibility using samples of known concentration [17]. Samples were tested in 8 duplicates with a single slide to assay intra-slide reproducibility and in 6 separate duplicates to assay inter-slide reproducibility.

\section{Results}

\subsection{Oriented immobilization of IgG for DHV1 detection}

Fig. 2 (5a) and (5b) showed the ellipsometric image of $200.0 \mathrm{ug} / \mathrm{ml}$ protein A layer. Relative to substrate, the net grayscale value was $4.9 \pm 1.3$. After combined with $\operatorname{IgG}$, the net grayscale value was changed to $41.7 \pm 3.4$ ( $4 \mathrm{a}$ and $4 \mathrm{~b}$ ). This demonstrated that IgG molecule was specifically interacted with protein A layer. DHV1 solution $\left(5 \times 10^{-6.5} \mathrm{LD}_{50} / \mathrm{ml}\right)$ was then applied with the protein $\mathrm{A}$ immobilized IgG layer and the net grayscale value is $69 \pm 4.1$ (3a and $3 \mathrm{~b})$. In order to acquire comparison between IgG direct immobilization and oriented immobilization, we also conducted 


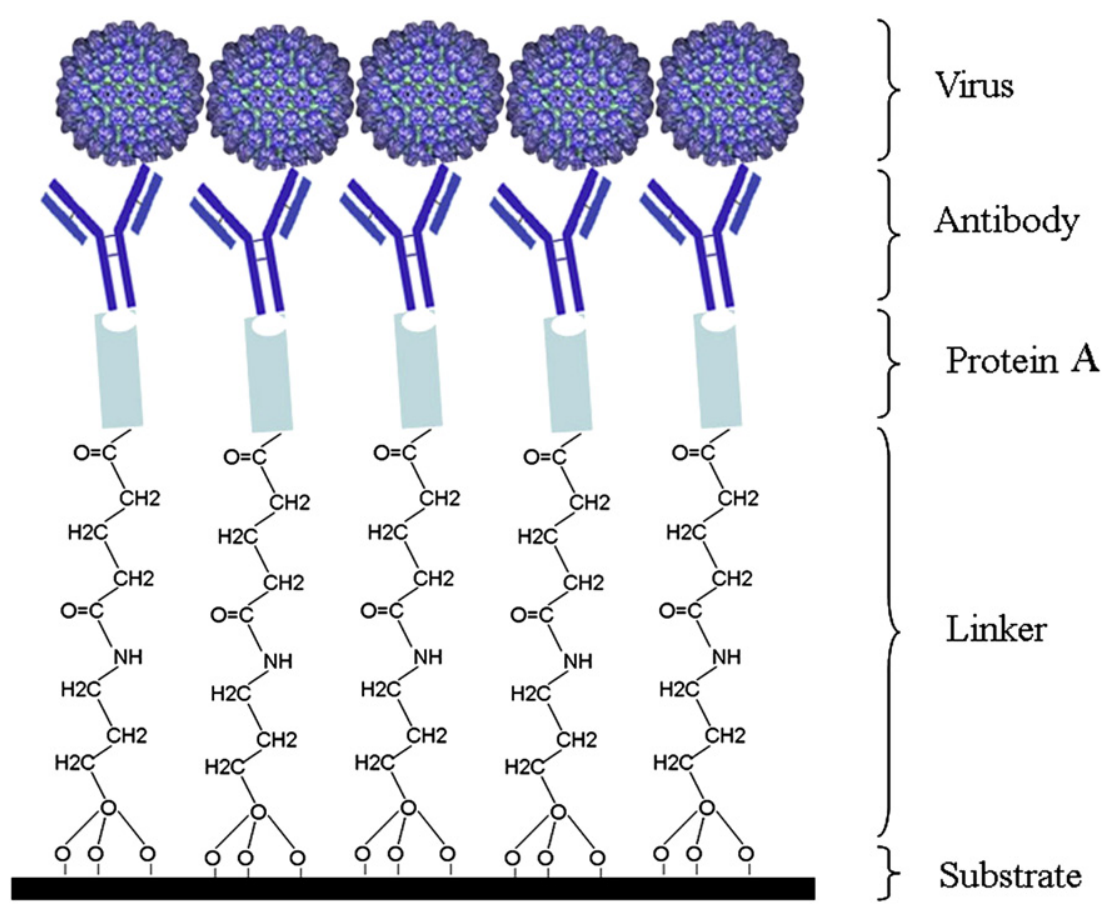

Fig. 1. Configuration of duck hepatitis virus detection by biosensor based on imaging ellipsometry.

IgG direct immobilization on the carboxyl surface. The net grayscale value relative to substrate is $55.3 \pm 4.9$ (2a and $2 b)$. After DHV1 exposed, the net grayscale value relative to IgG film layer was confined to $5.4 \pm 2.3$ ( $1 \mathrm{a}$ and $1 \mathrm{~b}$ ). Although the net grayscale value of direct immobilization of IgG was higher than that of oriented immobilization, the DHV1 combination was quite the contrary. Hence, oriented immobilization of IgG enhanced detection

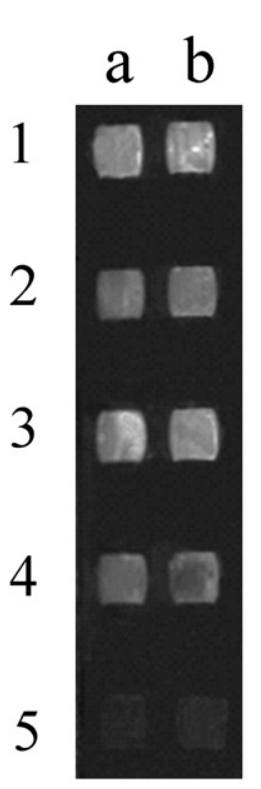

A

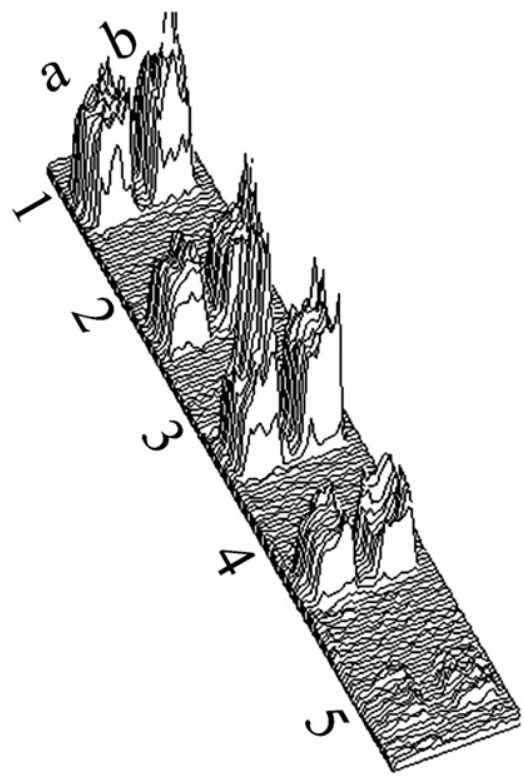

B
Fig. 2. Oriented immobilization of IgG for DHV detection (A) grayscale and (B) threedimension image. Dot 5 (protein A), 4 (protein A combined with $\operatorname{IgG}$ ), 3 (protein A combined with IgG and corresponding DHV), 2 (IgG directly immobilized on carboxyl surface) and 1 (combined with corresponding DHV). Dots are duplicates. performances of DHV1. The efficiency of antigen binding was increased about 2.4-fold by oriented immobilization.

\subsection{Multiple serotypes detection}

Most circumstances, we do not know which type of DHV1 was infected to ducks as multiple serotypes existence. It is very important for type-specific identification of the pathogen. In this study, total nine unit dots were manufactured in the form of $3 \times 3$ array. The procedures of oriented immobilization of IgGs and detection were strictly complied with the method above established. In brief, three dots in row1, IgG immobilized was anti-DHV1. Similarly, three units in row2 was anti-DHV1-g and in row3 was anti-DHV1-s. Antigen (DHV1, DHV1-g and DHV1-s) flowed sequentially through each row of the array (Fig. 3). Compared with dot $\mathrm{a} 2$ and $\mathrm{a} 3$ in row1, grayscale value of dot 1 was markedly higher than that of dot 2 and dot3. Accordingly, grayscale values of b1 and b3 are higher than b2, c2 and c3 higher than c1. The grayscale values of each dot were listed in Table 2 . That is to say, only corresponding antigen could interacts with its IgG. With this simple

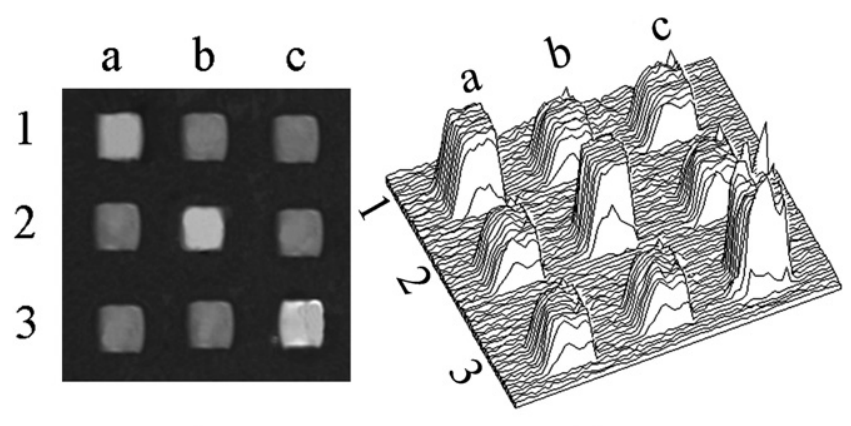

A

B

Fig. 3. Type-specific identification of DHV1, DHV1-g and DHV1-s (A) ellipsometric and (B) three-dimension image. Detailed descriptions can be seen in the text 
Table 2

Grayscale values in Fig. 5 protein dots.

\begin{tabular}{lllclc}
\hline No. & Grayscale & No. & Grayscale & No. & Grayscale \\
\hline a1 & 142.5 & a2 & 90.6 & a3 & 93.1 \\
b1 & 89.8 & b2 & 151.4 & b3 & 92.5 \\
c1 & 90.3 & c2 & 95.2 & c3 & 148.2 \\
\hline
\end{tabular}

layout, types of DHV1 were easily identified in a single experiment. When suspicious Duck Hepatitis was outburst, fluid from pleural cavity or chamber of heart can be applied for rapid identification by BIE in clinic laboratory. Therefore, it has obvious practicable value.

\subsection{Analytical sensitivity}

The analytical sensitivity was determined using five-fold serial diluted samples representing DHV1 type $\left(10^{-6.5} / 0.2 \mathrm{ml} 50 \%\right.$ lethal dose, $\mathrm{LD}_{50}$ ) as a reference for zero standard measurement within a same silicon wafer. The mean value of grayscale change (SD) determined by ten zero standard replicates was 1.2 , then, the corresponding grayscale value adding three standard deviations is 3.6. After transferred into virus titer, the minimum detection limit of DHV1 was as low as $8 \times 10^{-9.5} \mathrm{LD}_{50} / \mathrm{ml}$.

\subsection{Analytical specificity}

The specificity of the array was evaluated in the presence of avian influenza, duck plage virus, $R$. anatipestifer and $E$. coli, respectively (Fig. 4). The virus titer or bacteria content for above four species are $8 \log 2 \mathrm{HI}$ (Hemagglutination Inhibition) for $\mathrm{AI}$ (H9N2), $10^{3}$ CELD $_{50}$ (chicken embryo $50 \%$ lethal dose) for DPV, $2.1 \times 10^{7}$ for $R$. anatipestifer and $3.4 \times 10^{7} \mathrm{CFU} / \mathrm{ml}$ (colony formation unit $/ \mathrm{ml}$ ) for $E$. coli. Specificity for these pathogens was evaluated by calculation of the grayscale response. According to grayscale value changes of AI, DPV, GPV, R. anatipestifer, E. coli, healthy sera (negative control) and PBS (blank control), the percentage of grayscale response between these pathogen and DHV $\left(4 \times 10^{-6.5} \mathrm{LD}_{50} / 0.2 \mathrm{ml}\right)$ was calculated. The results were shown in Fig. 4. The grayscale values of the negative and blank control were both lower than the grayscale value of minimum content of DHV. This indicated that no false-positive results were obtained with the array with independent pathogens.

\subsection{Analytical reproducibility}

Protein biochips have intensively been investigated and developed for various diagnostic medical devices. Currently, there are no existing guidelines for the validation of antibody array. Standardized guideline would be important for reliable analysis. In this study, we consult some guidelines for protein chip evaluation [18]. The reproducibility was assessed by intra-slide and inter-slide measurements. CV\% values of intra-slide and inter-slide are $6.7 \%$, $5.8 \%, 7.4 \%$ and $15.2 \%, 13.6 \%, 11.9 \%$ for $4 \times 10^{-8.5}, 4 \times 10^{-7.5}$ and $4 \times 10^{-6.5} \mathrm{LD}_{50} / 0.2 \mathrm{ml}$, respectively (Table 3 ).

Table 3

Results of reproducibility analysis of BIE for DHV detection.

\begin{tabular}{llll}
\hline $\begin{array}{l}\text { Reference } \\
\text { sample }\end{array}$ & $\begin{array}{l}\text { Virus titer } \\
(\text { LD50) }\end{array}$ & $\begin{array}{l}\text { Intra-reproducibility } \\
(\text { CV\% })\end{array}$ & $\begin{array}{l}\text { Inter-reproducibility } \\
(\text { CV\% })\end{array}$ \\
\hline 1 & $4 \times 10^{-8.5}$ & $6.7(n=8)$ & $15.2(n=6)$ \\
2 & $4 \times 10^{-7.5}$ & $5.8(n=8)$ & $13.6(n=6)$ \\
3 & $4 \times 10^{-6.5}$ & $7.4(n=8)$ & $11.9(n=6)$ \\
\hline
\end{tabular}

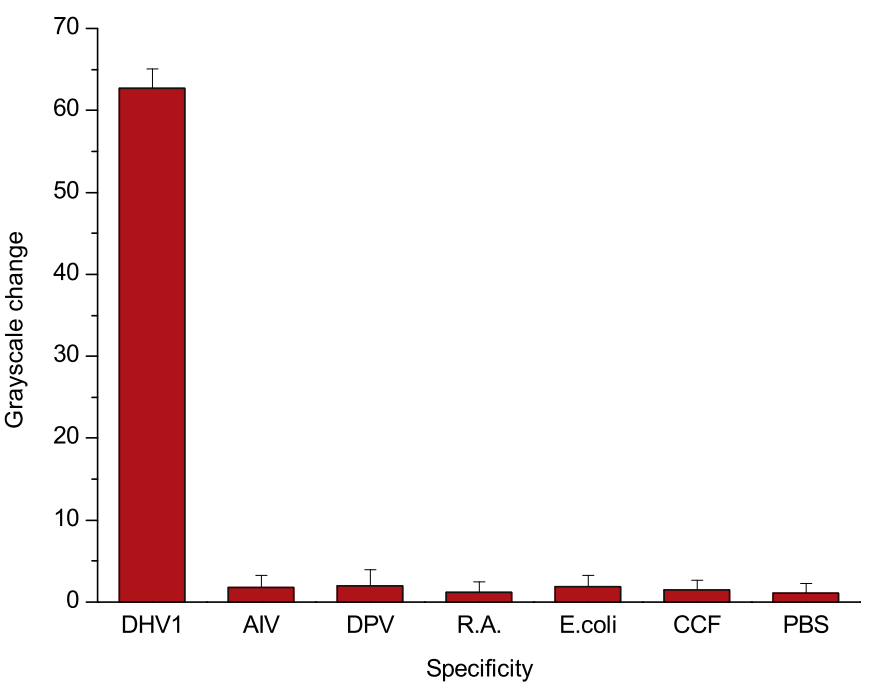

Fig. 4. Specificity.

\subsection{Application of clinic samples}

In order to explore the feasibility of BIE application for DHV1 detection, we investigated two clinic samples (DHV1, DHV1-g and DHV1-s) in parallel. The samples were collected from diseased ducklings with typical symptom. The pleural fluid was extracted and immediately measured by BIE. Fig. 5 displays typical grayscale image for DHV1, DHV1-g and DHV1-s detection. It was found that as the antigen titer increased, the brightness of the unit dots elevated. Changes in the grayscale value as a function of antigen titer were shown and three typical curves were obtained (Fig. 6). It was assumed that the antigen titers for DHV1-g and DHV1-s were $10^{-6.1} / 0.2 \mathrm{ml}$ and $10^{-5.8} / 0.2 \mathrm{ml} \mathrm{LD} 50$. Some stains observed of the

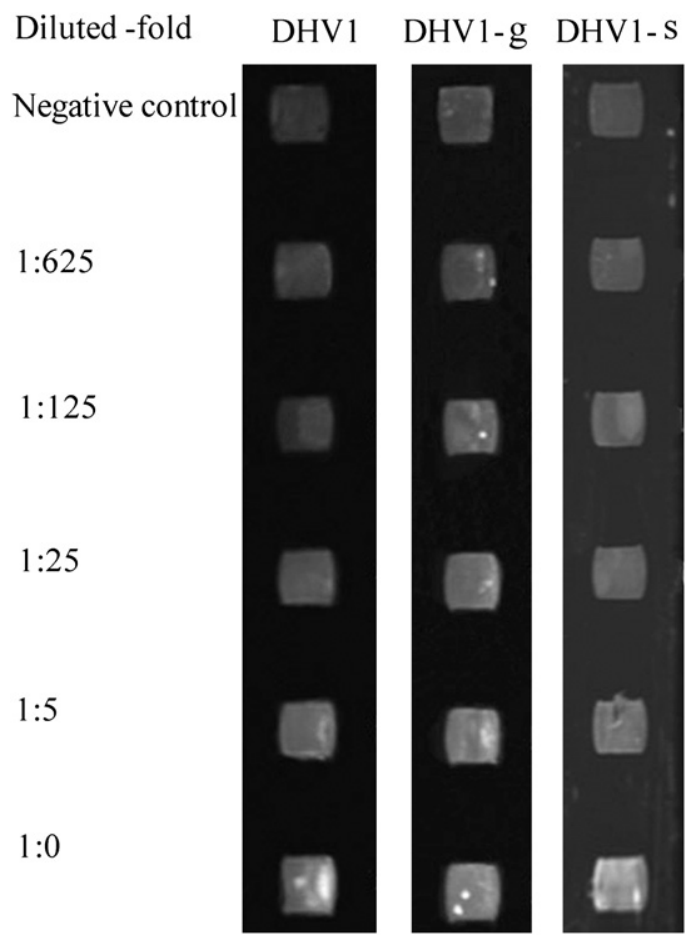

Fig. 5. Serial diluted clinic samples detected by BIE. Healthy duck serum was added for blank control. 


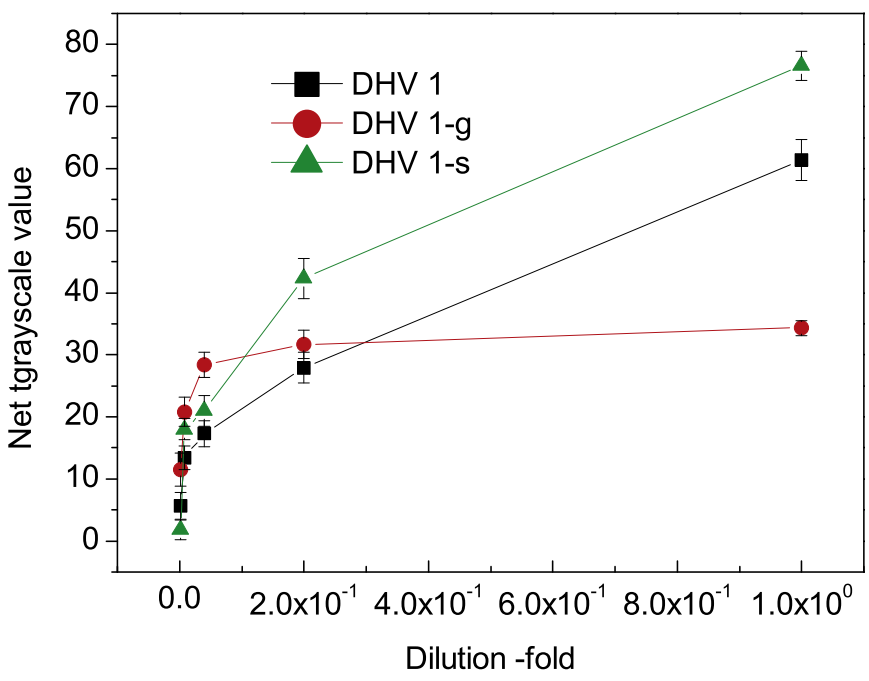

Fig. 6. Clinic sample application. DHV1, DHV1-g and DHV1-s were detected in parallel. Data are representative of 3 independent experiments for each type.

ellipsometric image might be caused by salt residuals or antigen aggregation.

\section{Discussion}

In this study, IgG could be directly immobilized on carboxyl surface through covalent attachment. The fashion of random arrangement makes no contributions to antigen-antibody interaction. The manner of oriented immobilization makes the Fc fragment stretching out of the surface and favor for antigen accessible. The grayscale changes for oriented immobilization of IgG were lower than direct immobilization of IgG on carboxyl surface, while grayscale changes after virus combination by oriented immobilization were higher than that by direct immobilization. This is completely consistent with the result of insulin application [19]. Imaging ellipsometry was used for thin layer analysis with high resolution to sub-nanometer level in vertical. Virus captured by antibody layer can be easily realized for imaging observation. Imaging ellipsometry has obvious grayscale response even the content of virus is confined to $8 \times 10^{-9.5} \mathrm{LD}_{50} / \mathrm{ml}$. Thus, the method has higher sensitivity.

In many cases, laboratories need more rapid and less cumbersome methods for the direct identification of the virus. At current, many methods can be used for detection of antigen, such as immunohistochemistry, viral isolation, and immuno-electron microscopy. These techniques are unable to detect antigen quickly due to requiring expensive equipment and trivial operations. The biosensor provides a platform that is capable of mass screening of clinical specimens. Besides above advantages, the BIE has merits as follows: first, using array, multiple unit dots can be fabricated and multiple samples can be simultaneously detected on a same substrate. With simple array layout, type-specific identification can also be achieved in a single experiment. The array also can be fabricated in $8 \times 6$ format with 48 unit dots even more than that. In this way, high throughput can be performed. Second, the detection time is $30 \mathrm{~min}$ expended. Compared with the mentioned techniques with hours and days consumed, the detection time is very short. Third, the sample was only $20.0 \mu$ needed. Fourth, BIE system can be automated, which could greatly simplify manipulations. Finally, BIE system is progressing in miniaturization and already obtained agitating fruit [20]. Thus, the proposed assay possesses considerable practical values.

\section{Acknowledgement}

The authors gratefully acknowledge financial support from Ministry of Science and Technology of China (2008AA02Z419), Natural Science Foundation $(10804083,20845003)$ of China, Chinese Academy of Sciences (KJCX2.YW.M04), Key Laboratory of Microgravity, Institute of Mechanics, CAS, and Doctoral Fund of southwest University, Chongqing, China.

\section{References}

[1] The potential of duck hepatitis virus (DHV-1) stimulating the body weight gain and the effects of silymarin on it in duckling.

[2] M.-C. Kim, Y.-K. Kwon, S.-J. Joh, S.-J. Kim, C. Tolf, J.-H. Kim, H.-W. Sung, A.M. Lindberg, J.-H. Kwon, Recent Korean isolates of duck hepatitis virus reveal the presence of a new geno- and serotype when compared to duck hepatitis virus type 1 type strains, Arch. Virol. 152 (2007) 2059-2072.

[3] G. Liu, FeiWang, Z. Ni, T. Yun, B. Yu, J. Huang, J. Chen, Genetic diversity of the VP1 gene of duck hepatitis virus type I (DHV-I) isolates from southeast China is related to isolate attenuation, Virus Res. 137 (2008) 137-141.

[4] C.-H. Tseng, N.J. Knowles, H.-J. Tsai, Molecular analysis of duck hepatitis virus type 1 indicates that it should be assigned to a new genus, Virus Res. 123 (2007) 190-203.

[5] P.P. Levine, J. Fabricant, Ahitherto-undescribed virus disease of ducks in North America, Cornell Vet. 40 (1950) 71-86.

[6] P.P. Levine, M.S. Hofstad, Duck disease investigation, Annu. Rep. N Y State Vet. Coll. Ithaca, (1945) 55-56.

[7] N.S. Hobson, I. Tothill, A.P.F. Turner, Microbial detection, Biosens. Bioelectron. 11 (1996) 455-477.

[8] R.H. Hall, Biosensor technology for detecting micrological foodborne hazards, Microbes Infect. 4 (2002) 425-432.

[9] G. Jin, P. Tengvall, I. Lundstrom, H. Arwin, A biosensor concept based on imaging ellipsometry for visualization of biomolecular interaction, Anal. Biochem. 232 (1995) 69-72.

[10] Z.-H. Wang, Y.-H. Meng, P.-Q. Ying, C. Qi, G. Jin, A label-free protein microfluidic array for parallel immunoassays, Electrophoresis 27 (2006) 4078-4085.

[11] Z.H. Wang, G. Jin, A label-free multisensing immunosensor based on imaging ellipsometry, Anal. Chem. 75 (2003) 6119-6123.

[12] G. Jin, R. Jansson, H. Arwina, Imaging ellipsometry revisited: developments for visualization of thin transparent layers on silicon substrates, Rev. Sci. Instrum. 67 (1996) 2930-2936.

[13] G. Jin, Development of biosensor based on imaging ellipsometry, Phys. Stat. Sol. 205 (a) (2008) 810-816.

[14] C. Oi, X.-S. Tian, S. Chen, J.-H. Yan, Z. Cao, K.-G. Tian, G.F. Gao, G. Jin, Detection of avian influenza virus subtype H5 using a biosensor based on imaging ellipsometry, Biosens. Bioelectron. 25 (2010) 1530-1534.

[15] Cai Qi, W. Zhu, Y. Niu, H.G. Zhang, G.Y. Zhu, Y.H. Meng, S. Chen, G. Jin, Detection of hepatitis B virus markers using a biosensor based on imaging ellipsometry, J. Viral Hepat. 16 (2009) 822-832.

[16] R.H. Jacobson, Validation of serological assays for diagnosis of infectious diseases, Rev. Sci. Tech. 17 (1998) 469-526.

[17] N.R.L.H.W. Roh, Y.H. Cho, J.B. Jung, H.N. Chung, W.S. Yang, W.K. Lee, H.K. Lee, G.H. Ryu, Development of a guideline for protein chip evaluation as medical devices, Curr. Appl. Phys. 6S1 (2006) 261-265.

[18] A. Ardizzoni, B. Capuccini, M.C. Baschieri, C.F. Orsi, F. Rumpianesi, S. Peppoloni, C. Cermelli, M. Meacci, A. Crisanti, P. Steensgaard, E. Blasi, A protein microarray immunoassays for the serological evaluation of the antibody response in vertically transmitted infections, Eur. J. Clin. Microbiol. Infect. Dis. 28 (2009) 1067-1075.

[19] Y.M. Bae, B.K. Oh, W. Lee, W.H. Lee, J.W. Choi, Detection of insulin-antibody binding on a solid surface using imaging ellipsometry, Biosens. Bioelectron. 20 (2004) 895-902.

[20] Y. Luo, G. Jin, A compact imaging ellipsometer for label-free biosensor. in: J.V. Sloten, P. Verdonck, M. Nyssen, J. Haueisen (Eds.), 4th European Conference of the International Federation for Medical and Biological Engineering, Antwerp, Belgium (2008), pp. 1050-1052. 